\title{
Open Epidermoid Cysts of the Vocal Folds: Our Experience with Four Cases
}

\author{
Nupur K Nerurkar ${ }^{1}$, Ruchi Shah ${ }^{2}$
}

\begin{abstract}
Subepithelial cysts (SECs), sulci, and mucosal bridges are often found in association with one another, resulting in dysphonia due to disturbed vibratory characteristics of the vocal folds along with an incomplete glottic closure in most instances. ${ }^{1}$ Histologically, cysts may be epidermoid or mucous retention and both are challenging to tackle, when other lesions such as mucosal bridges and sulci are coexistent. Epidermoid cysts may occasionally be open to the airway, referred to then as open cysts. ${ }^{2}$ The infrequent diagnosis of open cysts has consequently resulted in a few articles discussing the management of this entity. In this article, we have discussed four cases of open cysts of the vocal fold, which we diagnosed over the last 3 years (2015-2017) with a discussion of their management along with a review of the literature. All four open cysts were situated on the left vocal fold, and two cases had coexistent lesions, in the form of a mucosal bridge or a sulcus.

Keywords: Hoarseness, Mucosal bridge, Open cyst, Sulcus, Voice therapy.

International Journal of Phonosurgery \& Laryngology (2019): 10.5005/jp-journals-10023-1169
\end{abstract}

\section{INTRODUCTION}

A glottic cyst is a benign well-encapsulated collection of fluid of varying consistency, typically found lying free in the superficial lamina propria (SLP) underneath the epithelial layer and thus referred to as a subepithelial cyst (SEC). ${ }^{1}$ Two types of cysts, mucous retention cyst and epidermoid cyst are found within the Reinke's space. Mucous retention cysts are often translucent and are lined with cuboidal or columnar epithelium, presumed to arise from obstructed mucus-producing glands. ${ }^{3}$ Epidermoid cysts contain epithelium or accumulated keratin. They may be congenital in origin (dysembryoplastic theory) or may occur secondary to vocal abuse and phonotrauma (traumatic theory). ${ }^{4}$ An open epidermoid cyst can be seen when an epidermoid cyst spontaneously ruptures and opens into the laryngeal lumen, but still retains some of the accumulated keratin; in this case, the cyst's outline may be more subtle, and it usually assumes an oval shape with the long axis oriented anteriorly and posteriorly (Fig. 1). An open cyst may also produce a mottled appearance ${ }^{5}$ (Fig. 2A). Ruptured cysts may also generate vocal fold pits or mucosal bridges. According to the theory of Bouchayer and Cornut (1985), a mucosal bridge arises from two apertures in a single epidermoid cyst, superior, and inferior since the mucosal bridge between the two apertures is always thick and hyperkeratotic. ${ }^{6}$ Mucosal bridges may cause dysphonia of varying degrees, especially when associated with other laryngeal lesions such as vocal cysts or sulci (Fig. 2B). They are difficult to detect even on stroboscopy, and their significance and management are fraught with controversy with no real consensus on its optimal management. A type III sulcus refers more to a localized area of depression, also called a focal pit. Hyperkeratosis is common near the deepest aspects of the sac or pocket. Some authors believe that this represents an open epidermal cyst. ${ }^{7}$ Whether this entity of keratin deposition in a sac is a ruptured cyst or an accumulation in a focal pit, remains debatable.

\section{Case Description}

\section{Case 1}

A 30-year-old female presented to our voice clinic with a history of hoarseness and vocal fatigue, which she had noticed during
${ }^{1}$ Bombay Hospital and Medical Research Centre, Voice and Swallowing Centre, Mumbai, Maharashtra, India

${ }^{2}$ Bombay Hospital and Medical Research Centre, Mumbai, Maharashtra, India

Corresponding Author: Nupur K Nerurkar, Bombay Hospital and Medical Research Centre, Voice and Swallowing Centre, Mumbai, Maharashtra, India, Phone: +91 9821034085, e-mail: nupurkapoor@yahoo.com

How to cite this article: Nerurkar NK, Shah R. Open Epidermoid Cysts of the Vocal Folds: Our Experience with Four Cases. Int J Phonosurg Laryngol 2019;9(1):17-20.

Source of support: Nil

Conflict of interest: None

her pregnancy, progressively worsening over the last 2 years. She had a low-pitched and husky voice that sounded masculine. Stroboscopy revealed bilateral thick vocal folds with cheesy debris

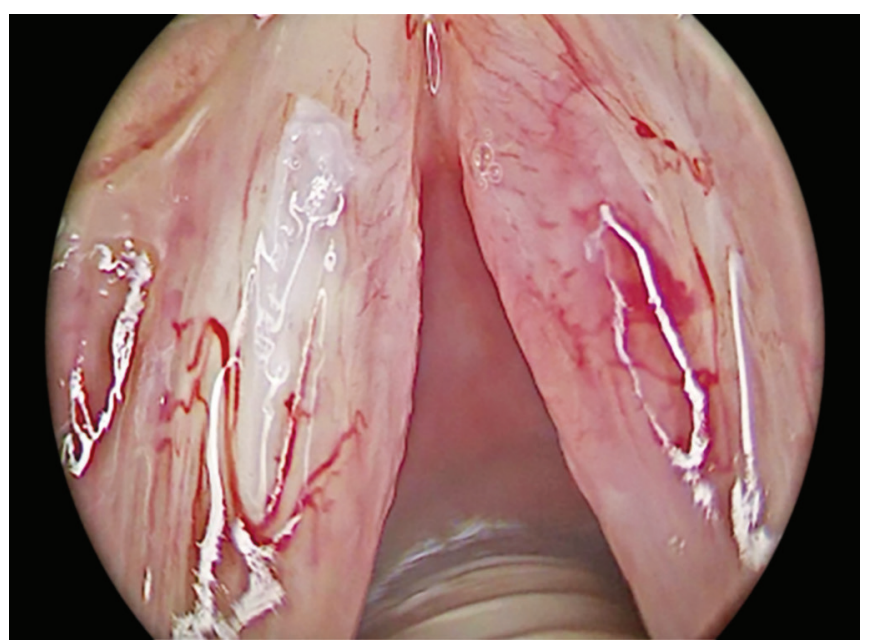

Fig. 1: Open cyst of the left vocal fold, which is oval in shape with its long axis oriented anteroposteriorly as seen in case 3. Reproduced from Nupur Kapoor Nerurkar, Textbook of Laryngology, Jaypee Brothers Medical Publishers (P) Ltd, 2017, Chapter 15-Fig. 27, with permission

o The Author(s). 2019 Open Access This article is distributed under the terms of the Creative Commons Attribution 4.0 International License (https://creativecommons. org/licenses/by-nc/4.0/), which permits unrestricted use, distribution, and non-commercial reproduction in any medium, provided you give appropriate credit to the original author(s) and the source, provide a link to the Creative Commons license, and indicate if changes were made. The Creative Commons Public Domain Dedication waiver (http://creativecommons.org/publicdomain/zero/1.0/) applies to the data made available in this article, unless otherwise stated. 
at the striking zone with a bilateral severely decreased amplitude of mucosal waves. Her modal frequency was $180 \mathrm{~Hz}$, with no range, and a maximum phonation time (MPT) was 6 seconds. Our clinical diagnosis on stroboscopy was bilateral open cysts with a possible left vocal fold sulcus. An endocrinology workup revealed hypothyroidism. The patient was started on thyroxin and also advised voice therapy. As her voice showed no improvement over the next 3 months, she was advised transoral microlaryngeal examination under general anesthesia with an informed consent taken for surgically excising the bilateral cysts and any other concurrent lesions if found appropriate. Intraoperatively a blunt flap elevator was used to palpate the vocal folds, which revealed a left vocal fold open cyst with an overlying thin mucosal bridge and a right vocal fold focal pit, also with an overlying mucosal bridge (Figs $2 \mathrm{C}$ to F). The left vocal fold open cyst was excised in to using a laser (AcuBlade) and sent for histopathological examination. The bilateral mucosal bridges were excised, and the entire epithelial lining of the right focal pit was made raw. Bilateral sutures were taken with 6-0 vicryl to approximate the new epithelial edges. The patient was advised voice rest for 10 days in the postoperative period and started on voice therapy on the 10th postoperative day. At the end of the first postoperative month, stroboscopy revealed early recovery of mucosal waves with a good closure pattern. Her modal frequency was $280 \mathrm{~Hz}$ with a range of $101-478 \mathrm{~Hz}$ and MPT of 13 seconds. The patient's vocal fatigue had improved remarkably. She has been advised to continue maintenance voice therapy.

\section{Case 2}

A 27-year-old obese female with a history of bariatric surgery done 2 years back presented with hoarseness and low-pitched voice since the age of 12 years. Her stroboscopy revealed a possible left vocal fold open cyst with a right vocal fold sulcus with a bilateral decreased mucosal wave. Her modal frequency was $205 \mathrm{~Hz}$ with a range of $200-240 \mathrm{~Hz}$ and MPT of 10 seconds. The patient was advised preoperative voice therapy for 1 month, followed by transoral microlaryngeal examination and surgery. Intraoperative palpation with a blunt flap elevator confirmed a left vocal fold elliptical open cyst and a right vocal fold incomplete mucosal bridge overlying a layer of subepithelial fibrosis. An incomplete mucosal bridge, in our opinion, is an epithelial mucosal bridge that just stops short of opening along its medial edge. The left vocal fold open cyst was excised, using laser and the infraglottic epithelium was sutured to cover the raw surface with a 6-0 vicryl. The right vocal folds incomplete mucosal bridge had underlying subepithelial fibrosis, which was lasered, and the raw surfaces were sutured with 6-0 vicryl (Fig. 3). The patient was advised voice rest for 10 days, followed by voice therapy. After 3 months of therapy, the amplitude of the mucosal wave improved significantly, and her modal frequency went up to $268 \mathrm{~Hz}$ with a range of $195-316 \mathrm{~Hz}$ and MPT of 14 seconds.

\section{Case 3}

A 20-year-old female presented with hoarseness since 6 months. Her stroboscopy revealed a left vocal fold open cyst and a right vocal fold SEC. Her modal frequency was $207 \mathrm{~Hz}$ with a range of $197-314 \mathrm{~Hz}$ and MPT of 6 seconds. The patient underwent transoral microlaryngeal surgery where on palpation intraoperatively, bilateral cysts were confirmed, and both of these cysts were leaking the cheesy material into the larynx, hence diagnosed as bilateral open cysts (Fig. 1). The cheesy material and the lining epithelium on the left and the right vocal fold cysts were removed with a laser. After removing all the ingrowing epithelium, the edges were sutured with 5-0 vicryl on the left and 6-0 vicryl on the right. The patient was advised voice rest post-surgery followed by voice therapy, and the results were remarkable in terms of vocal outcome with modal frequency coming up to $277 \mathrm{~Hz}$ and MPT of 14 seconds.
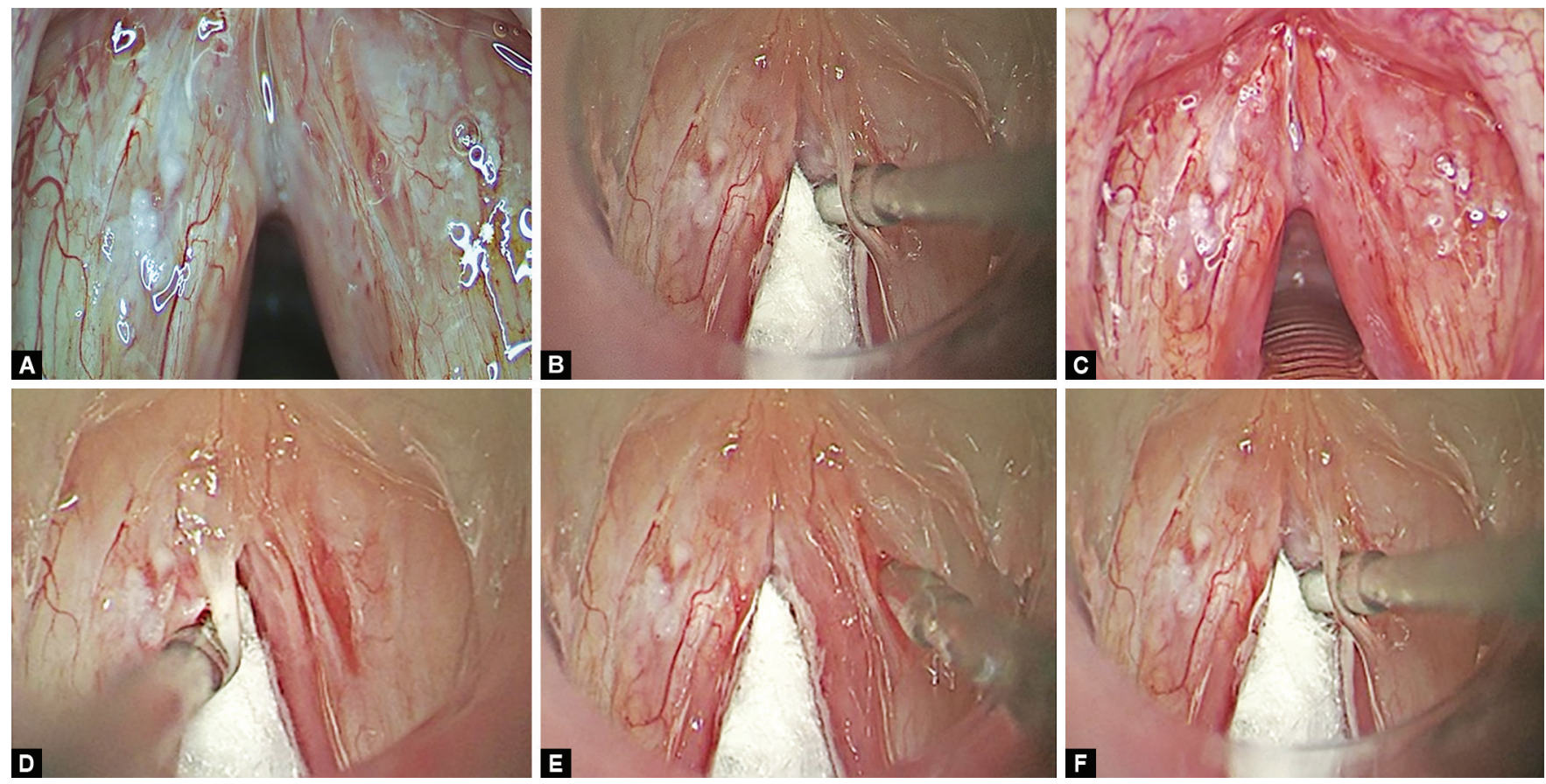

Figs $2 \mathrm{~A}$ to $\mathrm{F}$ : As seen in case 1 (A) Open cyst of the left vocal fold having a mottled appearance; (B) A thin epithelial mucosal bridge identified on the right vocal fold intraoperatively; (C) Left vocal fold open cyst along with (D) Left vocal fold mucosal bridge; (E) Right vocal fold focal pit; (F) Right vocal fold mucosal bridge 

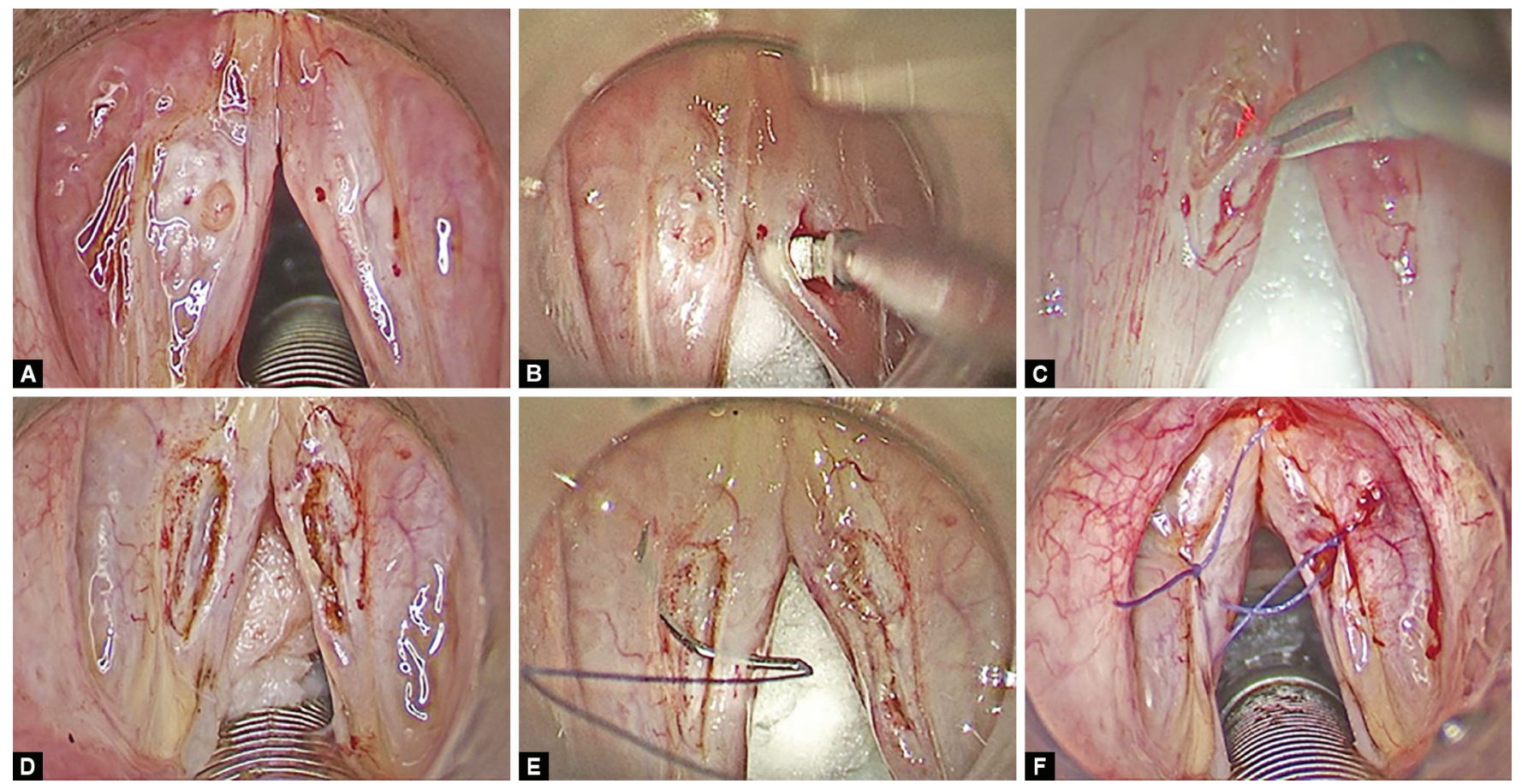

Figs 3A to F: Case 2: (A) Left vocal fold open cyst with (B) Right vocal fold incomplete mucosal bridge; (C) Laser excision of the left vocal fold open cyst; (D) After laser excision of the left vocal fold open cyst and subepithelial fibrosis under the right vocal fold incomplete mucosal bridge; (E) New epithelial edges being sutured using 6-0 vicryl; (F) Raw surfaces covered to give a smooth medial vibrating edge

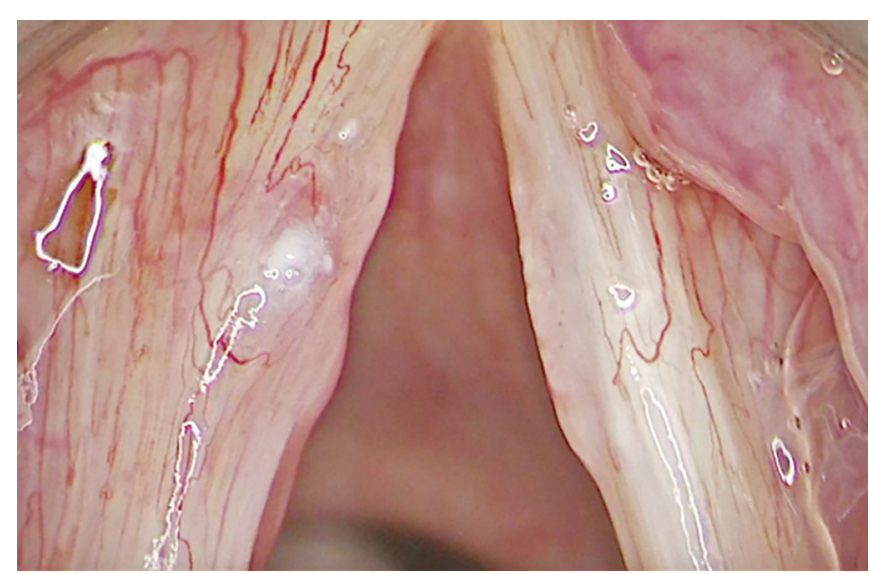

Fig. 4 : Case 4: Left vocal fold open cyst

\section{Case 4}

A 40-year-old housewife presented with hoarseness since 3 years. The patient was previously operated for a left vocal fold cyst 2 years back at another center. On stroboscopy, a left vocal fold open cyst was diagnosed with a right vocal fold contact lesion. Her modal frequency was $209 \mathrm{~Hz}$ with a range of 131-466 and MPT of 10 seconds. Intraoperatively on palpation, the left vocal fold open cyst was confirmed (Fig. 4). Laser excision of the cyst with the right vocal fold contact lesion was performed. The patient was given voice rest post-surgery followed by voice therapy, and her modal frequency increased to $270 \mathrm{~Hz}$ and MPT up to 16 seconds after 2 months.

\section{Discussion}

Vocal fold cysts are a common cause of dysphonia and are relatively easy to identify on stroboscopic evaluation and mostly require surgical excision. However, significant confusion exists regarding the diagnosis and management of open cysts particularly more so when they are coexisting with other vocal fold lesions such as mucosal bridges and sulci. One hypothesis claims that these lesions are the result of congenital anomalies occurring in the fourth and sixth branchial arch in the course of development of the larynx which could explain the finding that cysts, sulci, and mucosal bridges are seen together, with each of these entities representing a different stage in the evolution of the same congenital entity. ${ }^{8}$

An open cyst can often appear as a keratotic lesion on white light or stroboscopic evaluation/narrow-band imaging (NBI), and the clinical diagnosis is confirmed only after palpation of the lesion under suspension laryngoscopy under general anesthesia. Open cyst is a cyst which has burst into the laryngeal lumen as seen by a point opening in the cyst wall with extrusion of its content into the laryngeal lumen and thus may be differentiated from a focal pit, which is a localized area of depression with no overlying epithelial cover and has accumulated debris within. Mucosal bridges are also best picked up during microlaryngoscopy (MLS). Whenever two linear sulci are observed on stroboscopy or during MLS, a mucosal bridge should be palpated for. Whenever a patient is subjected to MLS, palpation with a blunt elevator is advocated to confirm the presence of a mucosal bridge.

Over the last 3 years (January 2015-December 2017), we have operated on 115 cases of vocal fold cysts, of which 4 were diagnosed as open cysts (3.47\%). In our series of 4 cases of open cysts, all patients were females and had similar findings of an open 
cyst found on the left vocal fold with only one patient having bilateral open cysts. The rate of benign vocal fold pathology has been found to be disproportionately higher in females. ${ }^{9}$ This result has been explained based on the higher fundamental frequency and consequently increase in collision trauma. ${ }^{10,11}$ In addition, less hyaluronic acid in the SLP of females is another possible cause. ${ }^{12}$ We could not find a possible explanation as to the location of all four cysts involving the left vocal fold. All four patients were presented with hoarseness of long-standing duration, probably suggesting its congenital etiology. Stroboscopic picture was suggestive of an open cyst in all four cases, and the findings were confirmed when these patients underwent MLS. Under suspension laryngoscopy, palpation of the vocal fold lesion is imperative. A blunt flap elevator was used in all cases to palpate both the vocal folds, which revealed open cysts, occasionally along with mucosal bridges, focal pits, and sulci on ipsilateral as well as contralateral vocal fold. Concurrent ipsilateral open cyst and mucosal bridge may be a result of variable openings made in two epidermoid cysts of the same vocal fold. Once a clinical diagnosis is made, surgical excision of these lesions poses a challenge. In all the cases, the open cyst was excised along with the entire cyst wall using a laser and sent for histopathological diagnosis. According to the histological classification (Koren classification) proposed by Jacob et al., ${ }^{13}$ all our cysts fall under type IV: Cysts lined by squamous epithelium with keratinization. The freshened edges were then sutured by $5-0$ or 6- 0 vicryl. As for the mucosal bridge, there exists a dichotomy of opinion among laryngologists as to the necessity to excise the mucosal bridge or let it be. We prefer its excision when it is thin and epithelial so as to avoid two epithelial surfaces vibrating as separate entities during phonation. However, thick mucosal bridges are best not removed for fear of decreasing the bulk of the vocal fold significantly.

In our series of four patients of open cysts, two had concurrent lesions. In the first case, the patient had bilateral complete thin mucosal bridges, which were excised, and the undersurface of the focal pit was made raw. In the second case, the patient had an incomplete mucosal bridge with underlying fibrosis, which was lasered. The most important step in all the cases was suturing of the new epithelial edges, which when sutured together brings the infraglottic epithelium to cover the medial vibrating edge. This ensures a smooth medial vibrating edge for a good postoperative vocal outcome. All patients were advised adequate voice rest after surgery so as to allow complete healing of the vocal folds followed by voice therapy for 2-3 months. The patients had a good vocal outcome 2 months after surgery with decreased vocal fatigue and an increase in the pitch of the voice. There was no voice deterioration in our small series of four cases. Postoperative recovery is found to be slower for vocal fold cysts that are on or near the vocal fold ligament compared to lesions in the subepithelial space. ${ }^{14}$ Similarly, the healing postoperatively in cases of open cysts was found to be more gradual than in the case of SECs. In our series, in cases with SEC, it took about 4-6 weeks for complete healing to occur whereas, in a cyst entering the vocal ligament (intraligamentous cysts) as well as open cysts, healing took a long time, taking up to 3-5 months. This was probably due to the larger surface area of the lesion and the use of microsutures to close the defect, which is known to result in an increased collagen concentration of lamina propria, which in turn attributes to vocal fold scarring and stiffness. ${ }^{15}$ It is advisable to counsel patients with suspected open vocal fold cysts regarding longer recovery time and intensive voice therapy postoperatively.

\section{Conclusion}

Over the last 3 years, $3.47 \%$ of our vocal fold cysts were diagnosed as open cysts. Out of the four cases, open cysts were found in association with mucosal bridges or sulci in two cases (50\%), and hence an accompanying lesion should always be looked and palpated for during surgery. Intraoperatively, after the excision of the cyst wall with its content, the new epithelial edges were sutured to cover the medial vibrating edge. The sutures may slow down the healing process but permit a smooth medial vibrating edge for a good vocal outcome postoperatively. The average improvement of MPT, in our series of patients, was 6 seconds with considerable improvement in the amplitude of the mucosal wave.

\section{References}

1. Nerurkar NK. Chapter 15: Cysts, sulci and mucosal bridge. Textbook of Laryngology. Jaypee Brothers Medical Publishers (P) Ltd; 2017. pp. 155-170.

2. Nerurkar NK. Chapter 5: Vocal fold cyst. Atlas of Phonomicrosurgery. Jaypee Brothers Medical Publishers (P) Ltd; 2018. pp. 28-44.

3. Natasha C, Khalid G. Chapter 25: Benign lesions of the larynx. Otorhinolaryngology- Head \& Neck Series (Laryngology). Thieme Medical \& Scientific Publishers Pvt Ltd; 2014. pp. 214-222.

4. Monday LA, Bouchayer M, Cornut G, et al. Epidermoid Cysts of the Vocal cords. Ann. Otol Rhinol Laryngol 1983;92(2 Pt 1):124-127. DOI: 10.1177/000348948309200205.

5. Open Epidermoid cyst. Laryngopedia by Bastian Medical Media. https://laryngopedia.com/open-epidermoid-cyst/. Accessed 10 September 2018.

6. Bouchayer M, Cornut G, Witzig E, et al. Epidermoid cysts, sulci and mucosal bridges of the true vocal fold: a report of 157 cases. Laryngoscope 1985;95(9 Pt 1):1087-1094.

7. Sataloff R, Chowdhary F. Chapter 22: Sulcus vocalis. Atlas of Endoscopic Laryngeal surgery. New Delhi: Jaypee Brothers Medical Publishers (P) Ltd; 2011. pp. 130-132.

8. Arnold GE. Dysplasia Dysphonia. Minor anomalies of the vocal cords causing persistent Hoarseness. Laryngoscope 1958;68(2):142-158. DOI: 10.1288/00005537-195802000-00006.

9. Herrington-Hall BL, Lee L, Stemple JC, et al. Description of laryngeal pathologies by age, sex, and occupation in a treatment-seeking sample. J Speech Hear Disord 1988;53(1):57-64. DOI: 10.1044/ jshd.5301.57.

10. Coyle SM, Weinrich BD, Stemple JC. Shifts in relative prevalence of laryngeal pathology in a treatment-seeking population. J Voice 2001;15(3):424-440. DOI: 10.1016/S0892-1997(01)00043-1.

11. Titze IR. Physiologic and acoustic differences between male and female voices. J Acoust Soc Am 1989;85(4):1699-1707. DOI: 10.1121/1.397959.

12. Schneider B, Bigenzahn W. Influence of glottal closure configuration on vocal efficacy in young normal-speaking women. J Voice 2003;17(4):468-480. DOI: 10.1067/S0892-1997(03)00065-1.

13. Jacob S, Rumelia K, Tuvia H, et al. Clinico pathologic study and classification of vocal cord cysts. Pathol Res Pract 2000;196(2):95-98. DOI: 10.1016/S0344-0338(00)80039-4.

14. Clark R, Blake S. Chapter 17: Vocal fold cyst and vocal fold fibrous mass. Operative Techniques in Laryngology. Springer Publications; 2008. pp. 109-112.

15. Rebecca C, Leandro L, et al. Histologic Comparison of vocal fold microflap healing with sutures and glue. Laryngoscope 2013;123(7):1709-1716. DOI: 10.1002/lary.23914. 